\title{
The dangers of drawing cohort profiles from period data: a research note
}

Alyson A. van Raalte ${ }^{1}$, Ugofilippo Basellini ${ }^{1,2}$, Carlo Giovanni Camarda ${ }^{2}$, Marília R. Nepomuceno ${ }^{1}$, Mikko Myrskylä ${ }^{1,3}$

${ }^{1}$ Max Planck Institute for Demographic Research (MPIDR), Rostock, Germany

${ }^{2}$ National Institute for Demographic Research (INED), Paris, France

${ }^{3}$ The University of Helsinki, Helsinki, Finland

Author emails: vanraalte@demogr.mpg.de; $\underline{\text { basellini@demogr.mpg.de; carlo-giovanni.camarda@ined.fr; }}$ nepomuceno@demogr.mpg.de; myrskyla@demogr.mpg.de

\begin{abstract}
Drawing cohort profiles and cohort forecasts from grids of age-period data is common practice in demography. In this research note, we: (1) estimate the bias in the cohort TFR and life expectancies calculated from such data, and (2) demonstrate that cohort Lee-Carter forecasts drawn from an ageperiod grid have implausible prediction intervals, especially when drawn from the upper and lower bounds of the time series parameter. These biases are surprisingly large, even when the cohort profiles are created from single-age, single-year period data. The danger is that we overinterpret deviations from expected trends, that in actual fact were induced by our own data manipulation.
\end{abstract}

Keywords: Lexis diagram, Lee-Carter, prediction intervals, lifespan variation, cohort fertility 


\section{Introduction}

As demographers, we are all aware that demographic events and person-years at risk can be split and aggregated into different formats on the Lexis diagram: age-period squares, cohort parallelograms, or for even more flexibility, Lexis triangles (Carstensen 2007; Keiding 1990; Wilmoth et al. 2019). But who among us has never created a cohort profile by 'taking the diagonals' of an ageperiod grid? Perhaps this is because most demographic data is formatted for period analysis. Possibly we wanted to analyze cohort trends from a methodology that was designed specifically to be used on an age-period grid. Whatever the reason, taking the diagonals is common practice (e.g. Kermack et al. 1934; Myrskylä et al. 2013; Preston and Wang 2006; Shkolnikov et al. 2011; Vogt et al. 2017), even when we suspect that it is not the best practice.

In this research note, we illustrate the dangers of drawing cohort profiles from period data using examples that we have recently encountered. The first example shows how artificial fluctuations in trends of mortality and fertility summary measures can arise when using the diagonals of age-period rates, due to differences in cohort size. The second example illustrates how completing cohort mortality from a period Lee-Carter mortality forecast can result in implausible prediction intervals for forecast cohort lifespan variation: there is less uncertainty for younger than older living cohorts.

It is well known that the larger the age-period square, the weaker the approximation of a real cohort. As the age-period square gets smaller, cohort patterns drawn from such squares start to approximate real (continuous-time) cohort lines. All of our empirical examples use single-year, singleage data and illustrate that the dangers do not vanish with this fine grid. The examples are fully reproducible ${ }^{1}$ : https://osf.io/xn5w7/?view only=223a3b90951043b1aada441b2b755225, using data from the Human Mortality Database (HMD 2022) and the Human Fertility Database (HFD 2022).

\footnotetext{
${ }^{1}$ Our Open Science Framework page is currently anonymous for peer review
} 


\section{Example 1: Cohort summary measures from period age-specific rates}

In Figure 1, we contrast cohort summary measures of fertility and mortality calculated from the two data formats: age-cohort parallelograms and the diagonals of age-period squares (see Figure A1 in the Appendix). The fertility example (left panel) presents trends in cohort completed fertility by age 40, also known as the cohort TFR, for Japanese females. Our mortality example (right panel) follows trends in cohort life expectancy at birth, cohort $e_{0}$, for Swedish females. Japan was chosen for the fertility example because of its rapid transition from high to low fertility, while Sweden was chosen for the mortality example because of its long time series of high-quality cohort data.

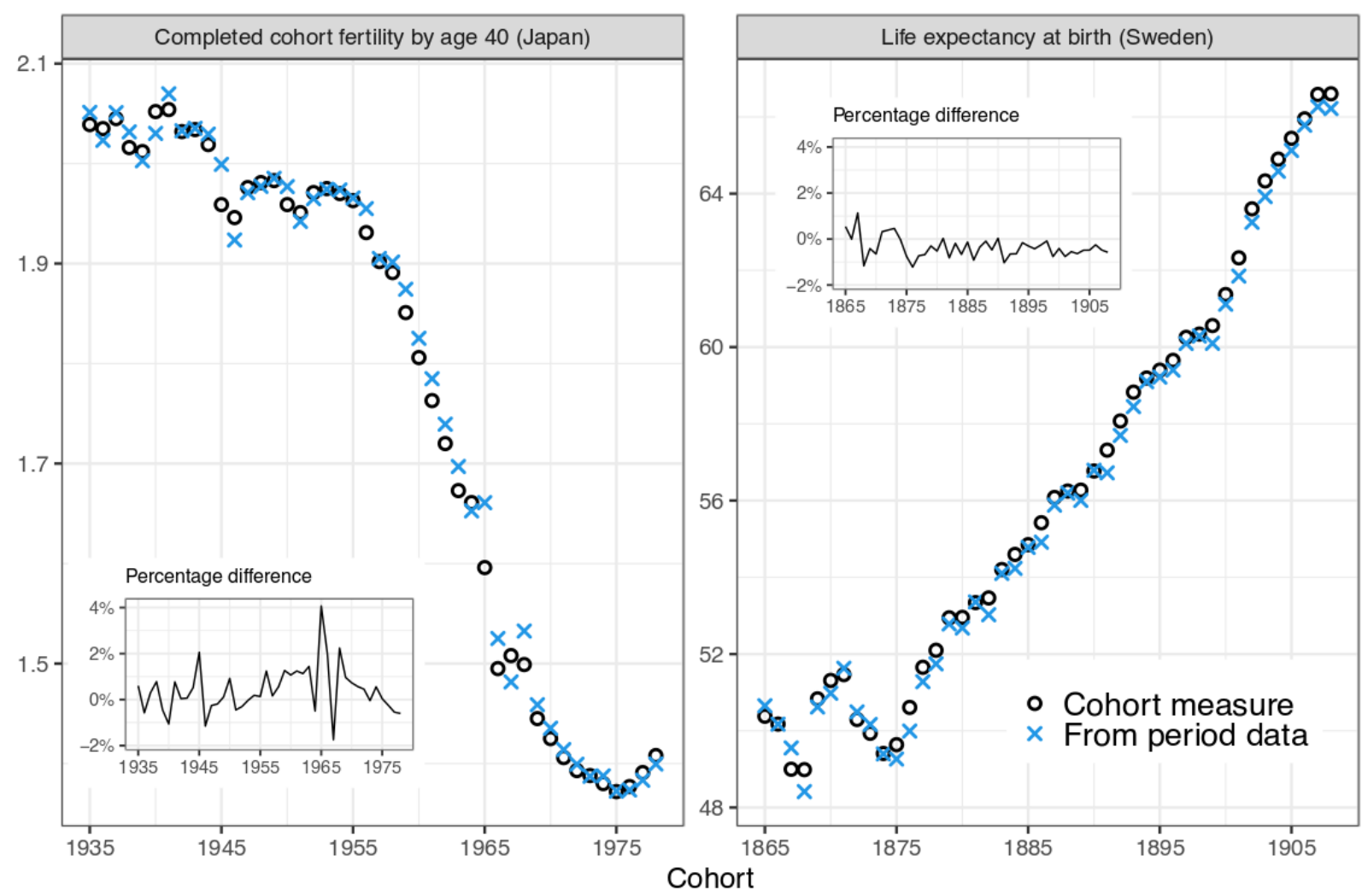

Figure 1: Completed cohort fertility by age 40 in Japan (left panel) and life expectancy at birth in Sweden (right panel) derived from cohort data (dots) and from diagonals of period data (crosses) for cohorts 1935--1978 and 1865--1908, respectively. The inset panels report the percentage differences between the two measures (cohort from period diagonal minus the true cohort). 
The trend in cohort TFR derived from the diagonals of age-period rates shows a number of unusual fluctuations, for instance around the 1966 birth cohort. This is only partially real. The true cohort trend is more stable. Changing cohort size explains the difference between the two trends. In 1965 there were 1.8 million births, which dropped to 1.4 million in 1966 (due to superstitious beliefs related to being born in the year of the "Fire-Horse" (Kaku 1975)) only to rebound to 1.9 million in 1967. Deriving summary cohort fertility measures from the age-period squares use rates drawn from events and exposures of the smaller 1966 cohort mixed with events and exposures from the larger neighbouring cohorts. These interactions cause an artificial fluctuation in the corresponding estimated cohort trends. The difference in cohort TFR between the two different data set-ups (cohort from period diagonals compared to the true cohort) is 0.8 percent on average (in absolute values), with a range of 1.7 to 4.1 percent. For 10 of the HFD countries with long series of fertility data ${ }^{2}$, the difference amounts to 0.6 percent on average (in absolute values), with a range of -2.1 to 4.1 percent.

Similarly, the cohort mortality example shows differences between the true cohort $e_{0}$ and the one approximated by the diagonals of period data. Altogether the difference in cohort $e_{0}$ calculated from the different data set-ups is 0.5 percent on average (in absolute values), with a range of -1.2 to 1.1 percent. A consistent underestimation of $e_{0}$ for the cohorts $1875--1908$ is evident from period data calculations. This underestimation is found in other HMD countries too: for 7 countries with a long series of mortality data ${ }^{3}$, the difference amounts to 0.7 percent on average (in absolute values), with a range of -4.8 to 2.7 percent.

\footnotetext{
2 Japan, France, Italy, the USA, Spain, Finland, Denmark, Sweden, the Netherlands, and England \& Wales.

${ }^{3}$ The same countries included in the TFR example, excluding Japan, the USA and Spain since these countries do not have mortality data for full cohorts. Moreover, for all countries we start the analysis from 1865 (or later, depending on data availability) to use more reliable historical data.
} 
Generally, we can expect underestimation of cohort TFR or $e_{0}$ derived from period data during contexts of rapid fertility or mortality decline, because the upper Lexis triangles are one-year earlier for the cohort from a period diagonal data set-up compared to a real cohort data set-up (see Figure A1).

\section{Example 2: Cohort lifespan variation drawn from period Lee-Carter forecasts}

There is a paucity of established methods for completing the mortality of partially observed cohorts, although there have been promising recent methodological developments (Basellini et al. 2020; Chiou and Müller 2009; Rizzi et al. 2021; Zanotto and Mazzuco 2017). As a result, it is not uncommon for researchers to derive cohort mortality forecasts by forecasting mortality from the period perspective and then taking the cohort diagonals to complete partially observed cohorts (Alburez-Gutierrez et al. 2021; Andreev and Vaupel 2006; Shkolnikov et al. 2011). Note that the death rates used for such a forecast could be conventional age-period rates for age $x$, time $t$, or age-cohort data where the death rates refer to age $x$ for cohort $t-x$.

We illustrate the perils of this approach with a Lee-Carter (1992) forecast ${ }^{4}$ of Swedish female mortality data. We forecast period age-specific mortality up to 2100 , based on period mortality rates from 1960 to 2019 . From these period forecasts, we completed cohort age-specific mortality profiles up to the 1980 birth cohort by taking the diagonals. These were used as inputs to cohort life tables from which we calculated two summary measures of longevity: cohort $e_{0}$ and cohort interquartile range, IQR. Figure 2 consists of the following: Panel A - estimated and forecast values of $k_{t}$ (the time index of

\footnotetext{
${ }^{4}$ The Lee Carter model expresses variation in death rates across age and time as follows: $\ln m_{\mathrm{x}, \mathrm{t}}=a_{\mathrm{x}}+b_{\mathrm{x}} k_{\mathrm{t}}+\varepsilon_{\mathrm{x}, \mathrm{t}}$, where $a_{\mathrm{x}}$ is the pattern of log mortality at age $x, k_{\mathrm{t}}$ is an index of the level of mortality at time $t, b_{\mathrm{x}}$ describes how much mortality at a given age changes with the overall mortality level changes, and $\varepsilon_{x, t}$ is the residual. In our results, following the standard LC estimation procedure, the model was fit to data from 1960 to 2019, $a_{\mathrm{x}}$ was set to equal the means over time of the $\ln m_{\mathrm{x}, \mathrm{t}}$, and the parameters $b_{\mathrm{x}}$ and $k_{\mathrm{t}}$ were estimated with singular value decomposition. An adjusted $k_{\mathrm{t}}$ (to match the observed number of deaths in each year) was forecast to decline linearly to 2100 using a random walk with drift.
} 
mortality) with 95\% prediction intervals corresponding to the upper and lower bounds of the simulated $k_{t}$ trajectories; Panel B - observed and forecast cohort life expectancy; Panel C-observed and forecast cohort IQR; and Panel D - a zoom on IQR values for the cohorts 1920-1980. While the $e_{0}$ trajectories appear reasonable, the IQR trajectories do not. The prediction intervals are implausibly larger for cohort 1943, which has been observed until age 76 , than for cohort 1957, which has only been observed until age 62. In fact, the upper and lower bounds of the prediction intervals cross over around cohort 1952, implying that there is no uncertainty about the point forecast for this partially observed cohort.

A

Time index of mortality $\left(\mathrm{k}_{\mathrm{t}}\right)$

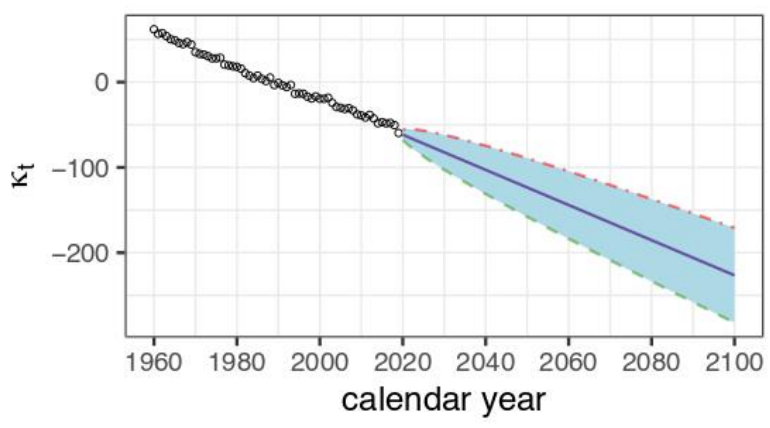

B Cohort life expectancy at birth $\left(e_{0}\right)$

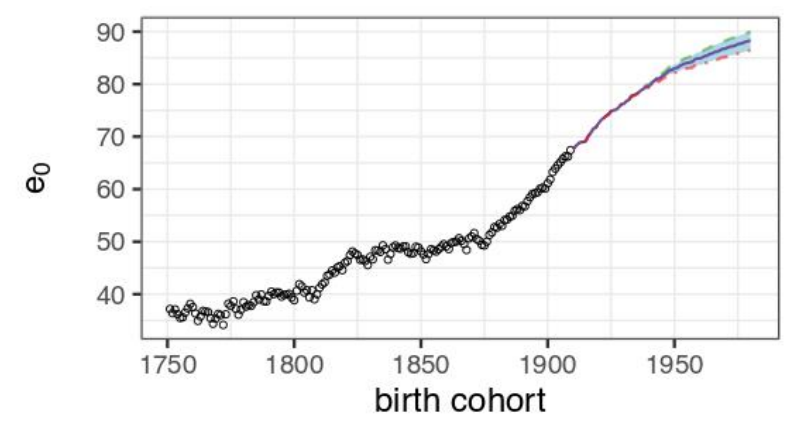

C Cohort lifespan inequality at birth (IQR)

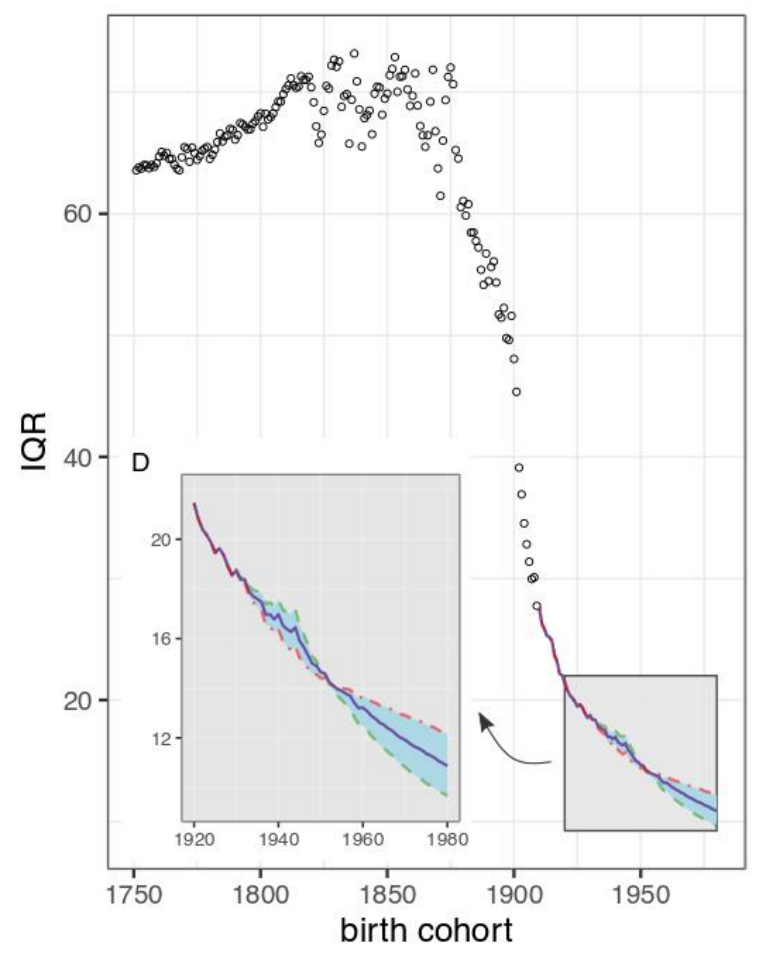

Figure 2: Panel A: Estimated $k_{t}$ for the years 1960--2019 (points) and forecast $k_{t}$ with $95 \%$ prediction intervals for the years 2020--2100 (lines) for Swedish females, ages 0-110+. The colours highlight the median, upper and lower percentile forecasts of $k_{t}$. Panel B: Observed (points) and forecast (lines) cohort life expectancy at birth, birth cohorts 1751--1980. Panel C: Observed (points) and forecast (lines) cohort interquartile range, birth cohorts 1751--1980. Panel D: Zoom in on the IQR forecasts for cohorts 1920--1980. 
The questionable prediction intervals that we found are a feature of a standard way to attribute uncertainty in the Lee-Carter model by taking the upper and lower bounds of the simulated $k_{t}$ trajectories. This method was suggested by Lee (2000) himself for period - not cohort - life table functions, and is widely implemented as the default method in popular statistical software packages (Hyndman et al. 2019; Villegas et al. 2018). The mapping of the uncertainty bounds of $k_{t}$ to the uncertainty bounds of period demographic summary measures such as life expectancy is appropriate, since they are generated from a single $k_{t}$ (Lee and Miller, 2001). However, for cohort life table measures, such as cohort lifespan variation, forecast age-specific death rates are generated from $k_{t}, k_{t+1}, k_{t+2}$, etc., and the associated complex relationship between their uncertainty bounds (Lee 2000). As a result, the simple mapping of the confidence bounds in $k_{t}$ to the confidence bounds in cohort lifespan variation become implausible.

The dynamics at play can also be interpreted demographically. Having a fixed age pattern of mortality decline parameter interacting with a linear mortality trend parameter will always produce the widening-narrowing-widening prediction intervals of panel $\mathrm{C}$. The narrowing of the prediction intervals arises around the forecast threshold age separating ages for which mortality decline acts to decrease lifespan variation from ages where mortality decline increases lifespan variation. To better illustrate the dynamics at play, we highlighted the forecast $k_{t}$ time series values corresponding to three relevant percentiles: median, lower and upper $95 \%$ prediction intervals. The lower forecast of $k_{t}$ results in steeper mortality decline compared to the median forecast. This compresses mortality at younger ages, causing forecast cohort lifespan variation to fall below the median forecast, and expands the age-atdeath distribution at older ages, causing forecast lifespan variation to rise above the median forecast. The opposite dynamic plays out for the upper percentile of the $k_{t}$ forecast.

These dynamics occur regardless of the chosen index of variation, the input data (age-period or age-cohort), or the population (Figures A2-A5 in Appendix). A potential solution is to calculate the 
lifespan measures for each simulated $k_{t}$ trajectory, and obtain the prediction interval from this distribution. Although these prediction intervals appear plausible, this approach has not been empirically tested whether the obtained uncertainty intervals are well calibrated.

\section{Discussion}

\section{Summary}

Drawing cohort profiles from age-period data or models can lead to patently false conclusions. In the first instance, using age-period data to calculate cohort $e_{0}$ and cohort TFR resulted in estimates that were on average 0.7 and 0.6 percent different from those calculated from age-cohort data, respectively. The largest deviations were a 4.1 percent difference in cohort TFR, and a -4.8 percent difference for cohort $e_{0}$. In the second example, drawing cohort profiles from Lee-Carter forecasts resulted in implausible prediction intervals for lifespan variability measures, when based on taking the upper and lower bounds of the $k_{t}$ time series parameter.

Should the artificial fluctuations in cohort fertility and mortality caused by the input data be considered large or small? To put this difference into perspective, the drop in period $e_{0}$ in either the United States or the United Kingdom from 2014 to 2017 was less than 0.1 percent (HMD 2021), which is seven times less than the average data-induced cohort life expectancy artifact. These declines have received extensive attention in the media, as well as in demographic and public health circles (Hiam et al. 2018; Ho and Hendi 2018). The steady drop in the US period TFR from 2007-2019, which has also generated considerable discussion (Guzzo and Hayford 2020; Hartnett and Gemmill 2020), was more on average around 1.8 percent per year (HFD 2021). In relative terms, the average data-driven artifact estimated for any single cohort TFR amounts to about one-third of the recent yearly drop in US period fertility. 
While the examples used related to fertility and mortality, these issues are equally pertinent in migration research. Migration flows fluctuate by year and season. In Europe, 1.3 million refugees, primarily from Syria, Iraq, and Afghanistan, claimed asylum in 2015 (Eurostat 2021). This was more than double the level seen in any year over the preceding decade. Two-thirds of these 2015 asylum seekers arrived in July or later that year. In a Lexis surface of the year of arrival ("year") and years since arrival ("age"), the late-2015 migration cohort would have some weight in "year 2015-age 0", heavy weight in square "year 2016-age 0", some weight in "year 2016-age 1", heavy weight in "year 2017-age1", etc., with the fluctuation continuing as this migration cohort is followed throughout its life course. An analysis of decisions on asylum seekers by year of arrival, based on square-based cohort inference, could be very different from decisions based on properly structured age-cohort data.

\section{Potential solutions to mitigate the problems:}

\section{Calculating cohort summary measures from period data}

National statistical agencies often provide data in aggregated age-period formats that are particularly suited to period analysis. When conducting cohort analyses, we should first split the data into Lexis triangles, and reshape it into cohort parallelograms. Methods to do so are well elaborated in Carstensen (2007). These methods have their assumptions, in particular, that births (or the event which defines the cohort) are evenly distributed at the beginning and end of the calendar year. This assumption is sometimes violated, for instance after exceptional events such as wars, famines, or epidemics (Aassve et al. 2020; Agadjanian and Prata 2002; Chandra et al. 2018; Lindstrom and Berhanu 1999); in anticipation of loss or gain in social benefits such as baby bonuses (Brunner and Kuhn 2011; Thévenon and Gauthier 2011); depending on cultural beliefs, for example, the period boost in fertility among Chinese accompanying dragon years (Goodkind 1991, 1995); or due to seasonal fluctuations such as the 2015 European migration example. In these cases, exposures can be distributed by month of birth 
or other event defining the cohort, as is now done for the exposure populations in the HMD and HFD (Wilmoth et al. 2019).

If the data we have on hand comes in the form of age-period rates and not the actual event and exposure counts, we envisage an adjustment of the rates which depends upon the changes in cohort sizes, if known.

\section{Completing cohort profiles from period forecasts}

The problem of the crossing prediction intervals is not a general feature of completing cohort mortality from period forecasts, but relates to an often-used approach of attributing uncertainty within the Lee-Carter forecasting methodology. Lee himself argued against using such an approach for cohort measures (Lee 2000), but never empirically elaborated upon the point, which remains common practice. We tested a few other period-based forecasting methodologies (see Figure A7 in the Appendix). Prediction intervals increased monotonically as expected across cohorts with more forecast ages for: (1) the relational STAD model which was designed to forecast changes to the shape of the period adult age at death distribution (Basellini and Camarda 2019); and (2) the CP-spline method which constrains mortality to lie within a range of plausible age profiles and time trends (Camarda 2019). However, just because the prediction intervals were increasing with younger cohorts, this does not mean they are correctly attributing uncertainty. Empirically, more testing would need to be done, specifically employing out-of-sample exercises to assess point and interval forecast accuracy, to see how closely the forecast uncertainty matches with the reality.

\section{Conclusion}

Drawing cohort profiles from period data and forecasts can lead to misleading conclusions including erroneous fluctuation in cohort summary measures, and implausible uncertainty in cohort 
forecasts. No one will be surprised that we find bias. But we expect many to be surprised by the magnitude of bias, even when cohort profiles and forecasts are drawn from a single-age, single-period grid. The danger is that we go down rabbit holes explaining deviations from expected trends, when the deviations themselves are an artefact of our own data manipulation.

We argue that cohort summary measures of fertility, mortality and migration should always be calculated on the basis of age-cohort data. Age-period data is easily transformed to Lexis triangles with available methods. In the case of cohort forecasting, crossing prediction intervals can be avoided by period-based forecasting methodologies that are not based on the Lee-Carter framework, or by simulation approaches within the Lee-Carter framework. However, we are of the opinion that uncertainty can only be properly accounted for with a cohort-based forecasting methodology. We end with a plea for researchers to further test and develop new cohort-based methods and models.

\section{References}

Aassve, A., Cavalli, N., Mencarini, L., Plach, S., \& Bacci, M. L. (2020). The COVID-19 pandemic and human fertility. Science, 369(6502), 370-371.

Agadjanian, V., \& Prata, N. (2002). War, peace, and fertility in Angola. Demography, 39(2), 215-231.

Alburez-Gutierrez, D., Kolk, M., \& Zagheni, E. (2021). Women's Experience of Child Death Over the Life Course: A Global Demographic Perspective. Demography, (9420770). https://doi.org/10.1215/00703370-9420770

Andreev, K. F., \& Vaupel, J. W. (2006). Forecasts of cohort mortality after age 50. MPIDR Working Paper WP 2006-012, 12, 2006. https://www.demogr.mpg.de/papers/working/wp-2006-012.pdf

Basellini, U., \& Camarda, C. G. (2019). Modelling and forecasting adult age-at-death distributions. Population studies, 73(1), 119-138.

Basellini, U., Kjærgaard, S., \& Camarda, C. G. (2020). An age-at-death distribution approach to forecast cohort mortality. Insurance: Mathematics and Economics, 91, 129-143. https://doi.org/10.1016/j.insmatheco.2020.01.007

Brunner, B., \& Kuhn, A. (2011). Financial Incentives, the Timing of Births, Birth Complications, and Newborns' Health: Evidence from the Abolition of Austria's Baby Bonus. SSRN Electronic Journal. https://doi.org/10.2139/ssrn.1963809

Camarda, C. G. (2019). Smooth constrained mortality forecasting. Demographic Research, 41, 10911130. https://doi.org/10.4054/DemRes.2019.41.38

Carstensen, B. (2007). Age-period-cohort models for the Lexis diagram. Statistics in Medicine, 26(15), 
3018-3045.

Chandra, S., Christensen, J., Mamelund, S.-E., \& Paneth, N. (2018). Short-term birth sequelae of the 1918-1920 influenza pandemic in the United States: state-level analysis. American Journal of Epidemiology, 187(12), 2585-2595.

Chiou, J.-M., \& Müller, H.-G. (2009). Modeling Hazard Rates as Functional Data for the Analysis of Cohort Lifetables and Mortality Forecasting. Journal of the American Statistical Association, 104(486), 572-585. https://doi.org/10.1198/jasa.2009.0023

Eurostat (2021). Asylum statistics [Data set]. https://ec.europa.eu/eurostat/statisticsexplained/index.php?title=Asylum_statistics. Accessed 5 May 2021

Goodkind, D. M. (1991). Creating New Traditions in Modern Chinese Populations: Aiming for Birth in the Year of the Dragon. Population and Development Review, 17(4), 663.

Goodkind, D. M. (1995). The Significance of Demographic Triviality: Minority Status and Zodiacal Fertility Timing Among Chinese Malaysians. Population Studies, 49(1), 45-55.

Guzzo, K. B., \& Hayford, S. R. (2020). Pathways to parenthood in social and family contexts: Decade in review, 2020. Journal of Marriage and Family, 82(1), 117-144.

Hartnett, C. S., \& Gemmill, A. (2020). Recent trends in US childbearing intentions. Demography, 57(6), 2035-2045.

HFD (2022). Human Fertility Database [Data set]. Max Planck Institute for Demographic Research (Germany) and Vienna Institute of Demography (Austria). Available at www.humanfertility.org. Data downloaded on 15 September 2021.

Hiam, L., Harrison, D., McKee, M., \& Dorling, D. (2018). Why is life expectancy in England and Wales 'stalling'? Journal of Epidemiology and Community Health, 72(5), 404-408.

HMD (2022). Human Mortality Database [Data set]. University of California, Berkeley (USA), and Max Planck Institute for Demographic Research (Germany). Available at www.mortality.org or www.humanmortality.de. Data downloaded on 15 September 2021.

Ho, J. Y., \& Hendi, A. S. (2018). Recent trends in life expectancy across high income countries: retrospective observational study. BMJ, 362:k2562. https://doi.org/10.1136/bmj.k2562

Hyndman, R., Booth, H., Tickle, L., \& Maindonald, J. (2019). Demography: Forecasting mortality, fertility, migration and population data. $R$ package version 1.22 .

Kaku, K. (1975). Increased induced abortion rate in 1966, an aspect of a Japanese folk superstition. Annals of Human Biology, 2(2), 111-115.

Keiding, N. (1990). Statistical inference in the Lexis diagram. Philosophical Transactions of the Royal Society of London. Series A: Physical and Engineering Sciences, 332(1627), 487-509.

Kermack, W. O., McKendrick, A. G., \& McKinlay, P. L. (1934). Death-Rates in Great Britain and Sweden. Some General Regularities and their Significance. Lancet, 698-703.

Lee, R. (2000). The Lee-Carter Method for Forecasting Mortality, with Various Extensions and Applications. North American Actuarial Journal, 4(1), 80-91.

Lee, R. D., \& Carter, L. R. (1992). Modeling and forecasting US mortality. Journal of the American Statistical Association, 87(419), 659-671.

Lee, R., \& Miller, T. (2001). Evaluating the performance of the Lee-Carter method for forecasting mortality. Demography, 38(4), 537-549.

Lindstrom, D. P., \& Berhanu, B. (1999). The impact of war, famine, and economic decline on marital fertility in Ethiopia. Demography, 36(2), 247-261.

Myrskylä, M., Goldstein, J. R., \& Cheng, Y. A. (2013). New cohort fertility forecasts for the developed world: Rises, falls, and reversals. Population and Development Review, 39(1), 31-56.

Preston, S. H., \& Wang, H. (2006). Sex mortality differences in the United States: The role of cohort smoking patterns. Demography, 43(4), 631-646.

Renshaw, A. E., \& Haberman, S. (2006). A cohort-based extension to the Lee-Carter model for mortality 
reduction factors. Insurance: Mathematics and Economics, 38(3), 556-570.

Rizzi, S., Kjærgaard, S., Bergeron Boucher, M.-P., Camarda, C. G., Lindahl-Jacobsen, R., \& Vaupel, J. W. (2021). Killing off cohorts: Forecasting mortality of non-extinct cohorts with the penalized composite link model. International Journal of Forecasting, 37(1), 95-104.

Shkolnikov, V. M., Jdanov, D. A., Andreev, E. M., \& Vaupel, J. W. (2011). Steep Increase in Best-Practice Cohort Life Expectancy. Population and Development Review, 37(3), 419-434.

Thévenon, O., \& Gauthier, A. H. (2011). Family policies in developed countries: a 'fertility-booster' with side-effects. Community, Work \& Family, 14(2), 197-216.

Villegas, A., Millossovich, P., Kaishev, V., \& Villegas, M.A. (2018). StMoMo: Stochastic Mortality Modelling. R package version 0.4.1.

Vogt, T., van Raalte, A., Grigoriev, P., \& Myrskylä, M. (2017). The German East-West Mortality Difference: Two Crossovers Driven by Smoking. Demography, 54(3), 1051-1071.

Wang, H., \& Preston, S. H. (2009). Forecasting United States mortality using cohort smoking histories. Proceedings of the National Academy of Sciences, 106(2), 393-398.

Wilmoth, J. R., Andreev, K., Jdanov, D., Glei, D., \& Riffe, T. (2019, October 6). Methods Protocol for the Human Mortality Database.

Zanotto, L., \& Mazzuco, S. (2017). Reconstruction of cohort data via EM algorithm. Paper presented at the XXVIII International Population Conference, Cape Town, South Africa. 


\section{Appendix}

In this Appendix, we show additional results of our analysis.

Figure A1 is a Lexis representation of the difference between properly structured age-cohort data, and approximating cohorts by 'taking the diagonals' of age-period data.

Figures A2-A7 report the results of our sensitivity analysis on example 2 of the manuscript. Figure A2 shows observed and forecast trends for two different indicators of lifespan variability, namely the life disparity and Gini concentration index. The figure shows that the crossing prediction intervals are not specific to the IQR measure, but rather a common feature of the lifespan variability indicators. However, it also appears that the overlap is somewhat reduced in the case of the Gini index. Figures A3 and A4 show the results focusing only on adult ages, i.e. starting the analysis from age 30 rather than age 0 . In Figure $A 3$, we follow the approach of the main analysis and we employ period data. In Figure A4, we start instead from raw cohort mortality data, we reshape it onto an age-period grid, forecast mortality and then derive the cohort measures from the diagonals of the Lexis diagram. In both cases, the figures show a greater overlap of the prediction intervals as compared to the analysis starting from age 0 . Moreover, Figure A5 replicates our analysis for French females. The crossing intervals are present also for this population. Figure A6 shows the forecast IQR obtained with the Lee-Carter model employing the standard and a simulation approach with simulated trajectories of $k_{t}$. The issue of crossing prediction intervals disappears when employing the latter approach. Finally Figure A7 compares the Lee-Carter model with the STAD and CP-splines approaches to forecast mortality. The figure highlights that the latter models do not display the crossing prediction intervals issue that is present in the Lee-Carter model. Both the STAD and CP-splines forecast lower reductions of the cohort IQR, with the former approach displaying larger prediction intervals than the latter. 


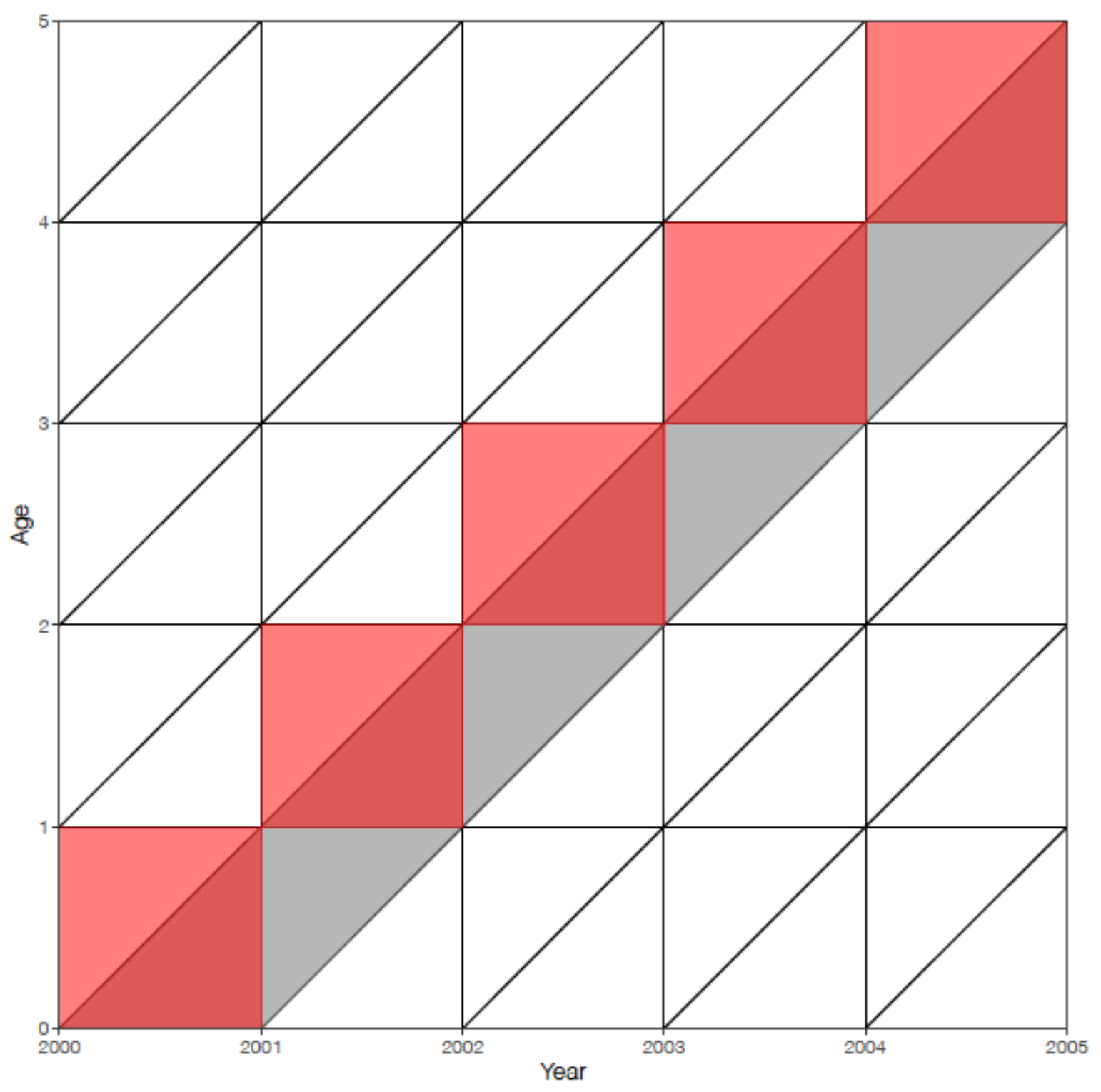

Figure A1: Lexis representation of the difference between the two data structures. Properly structured age-cohort data is represented in grey, and approximating cohorts by 'taking the diagonals' of ageperiod data is represented in red. The data from the upper Lexis triangles will always be one calendar year earlier for the age-period compared to age-cohort data. 


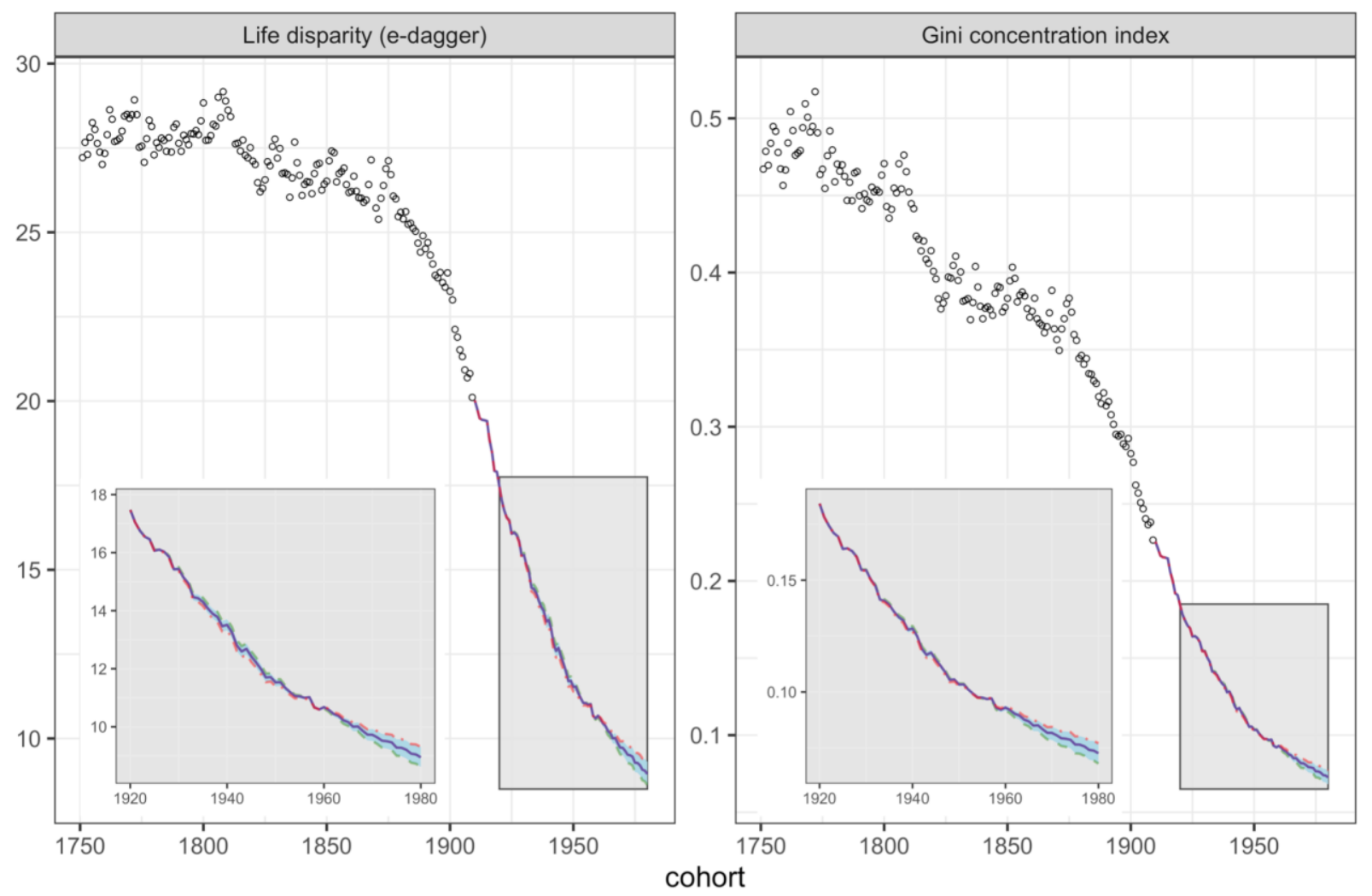

Figure A2: Observed (points) and forecast (lines) cohort lifespan variability measured with lifespan disparity (left panel) and Gini concentration index (right panel) for Swedish females, birth cohorts 1751-1980. The inset plot zooms in on the overlap of projected mortality patterns for cohorts 1920--1980. The prediction intervals are wider over cohorts in the 1930s and 1940s than those of the 1950s. 
A Time index of mortality $\left(\kappa_{\mathrm{t}}\right)$

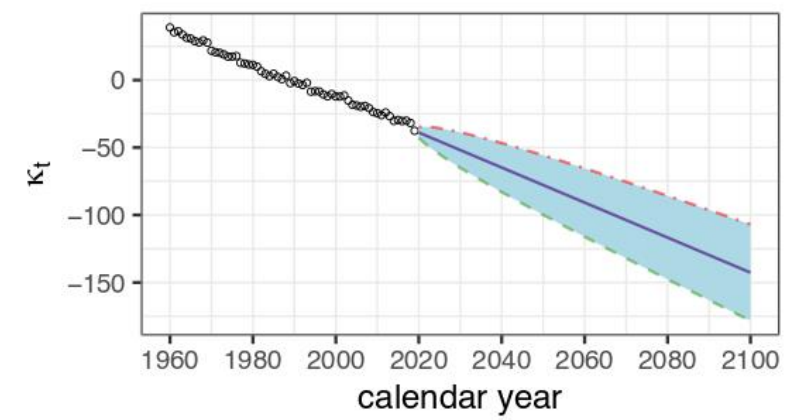

B

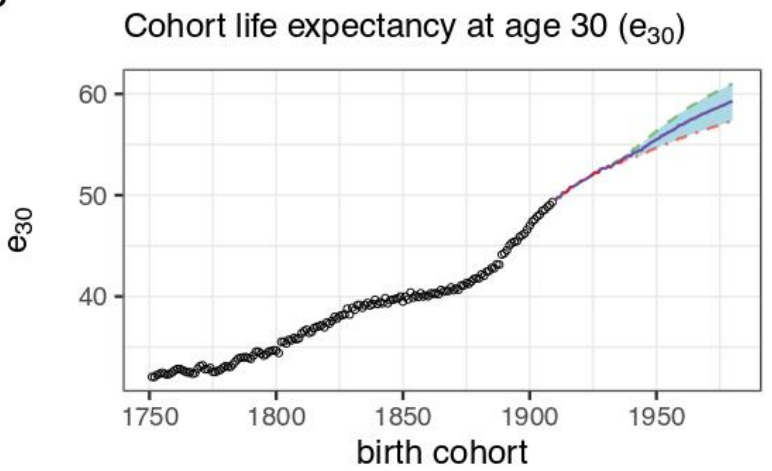

C Cohort lifespan inequality at age 30 (IQR)

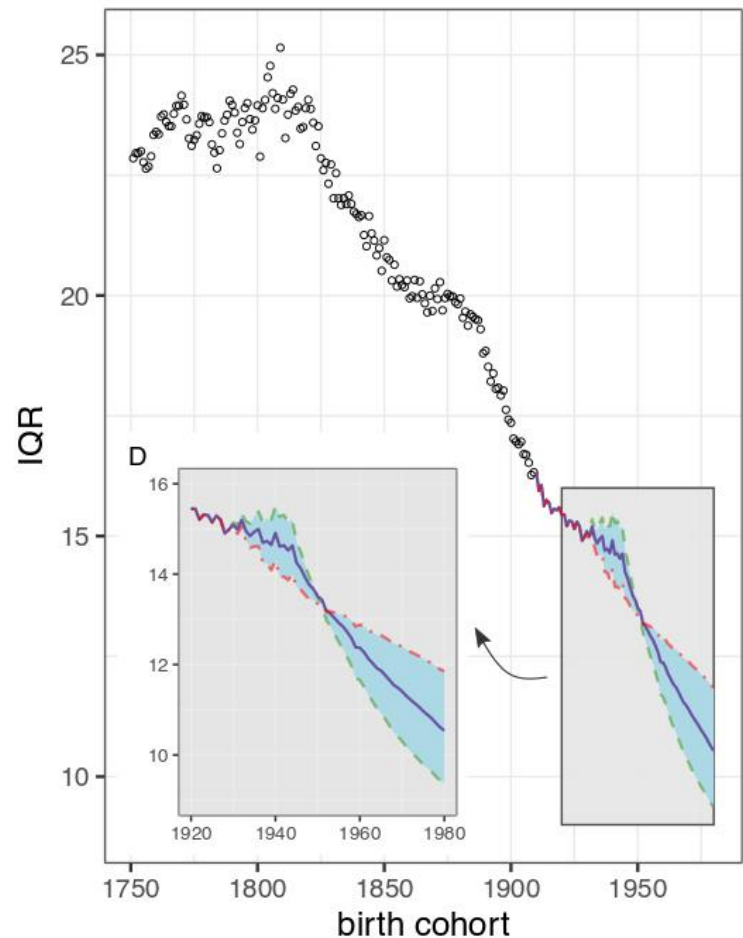

Figure A3: Panel A: Estimated $k_{t}$ for the years 1960--2019 (points) and forecast $k_{t}$ with 95\% prediction intervals for the years 2020--2100 (lines) for Swedish females, ages 30-110+. The colours highlight the median, upper and lower percentile forecasts of $k_{t}$. Panel B: Observed (points) and forecast (lines) cohort life expectancy at age 30, birth cohorts 1751--1980. Panel C: Observed (points) and forecast (lines) cohort interquartile range at age 30, birth cohorts 1751--1980. Panel D: Zoom in on the IQR forecasts for cohorts 1920--1980. 
A

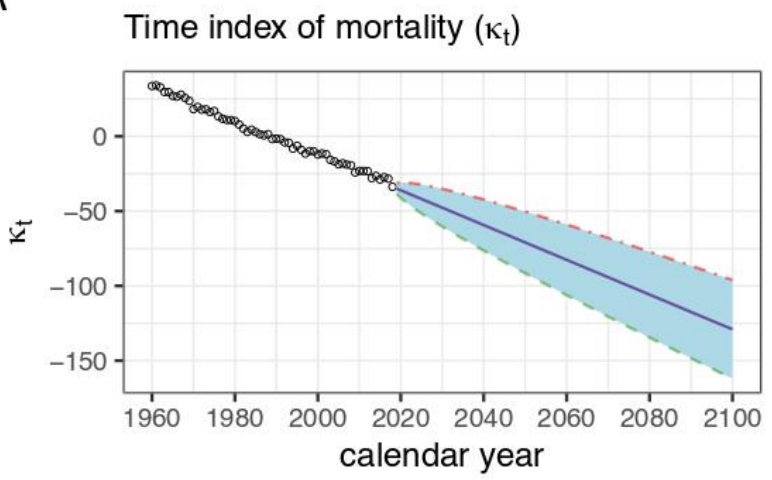

B

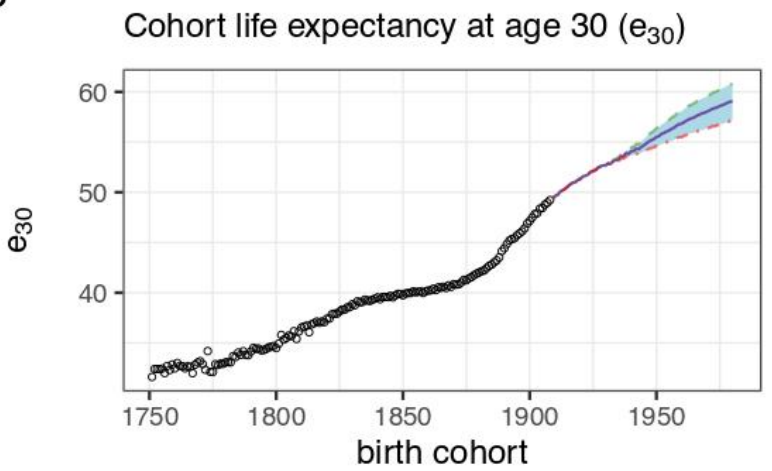

C Cohort lifespan inequality at age 30 (IQR)

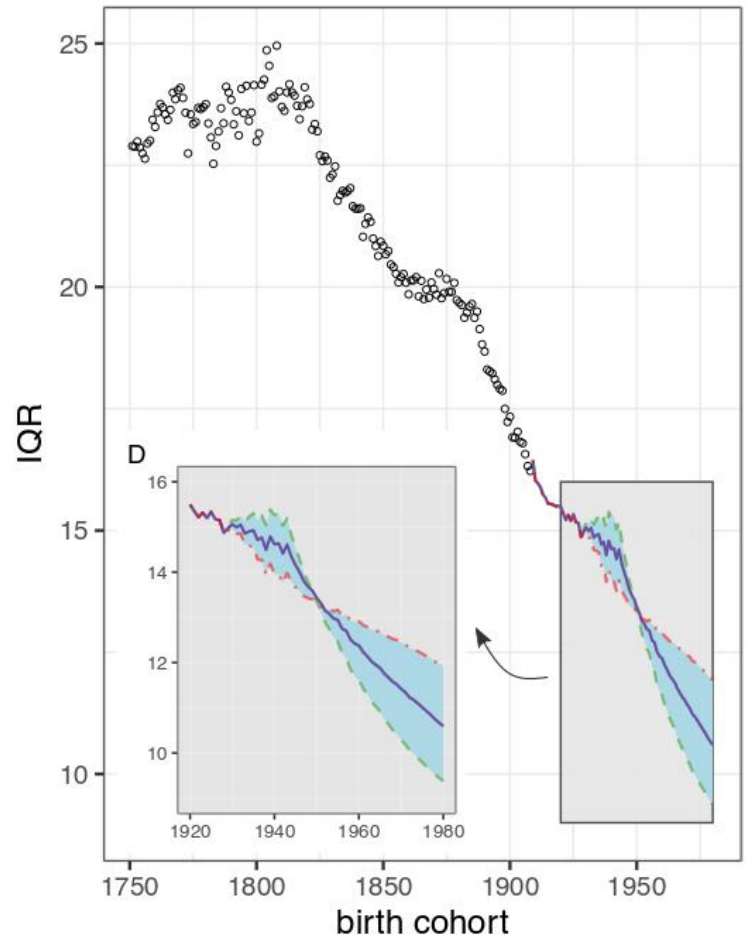

Figure A4: same analysis of Figure A3, employing cohort mortality data reshaped onto an age-period grid rather than period mortality data. 
A Time index of mortality $\left(\kappa_{\mathrm{t}}\right)$

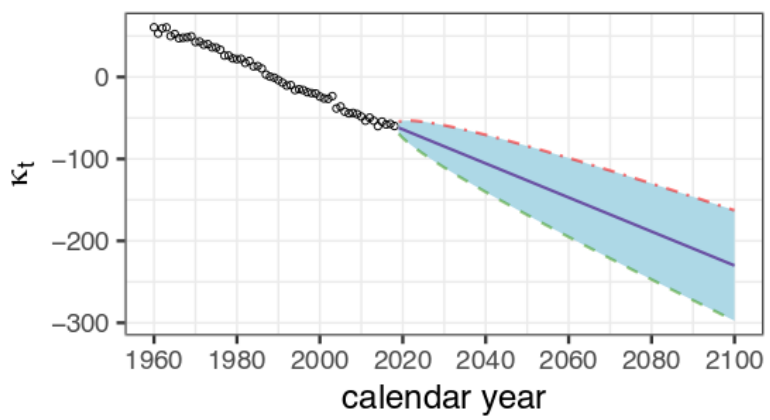

B

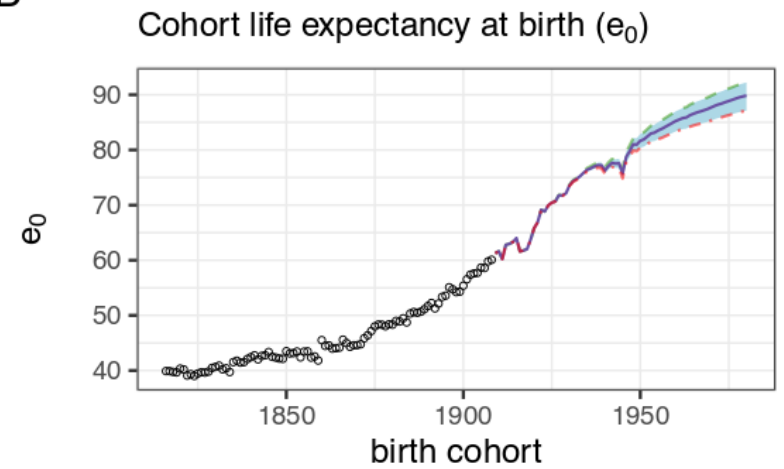

C Cohort lifespan inequality at birth (IQR)

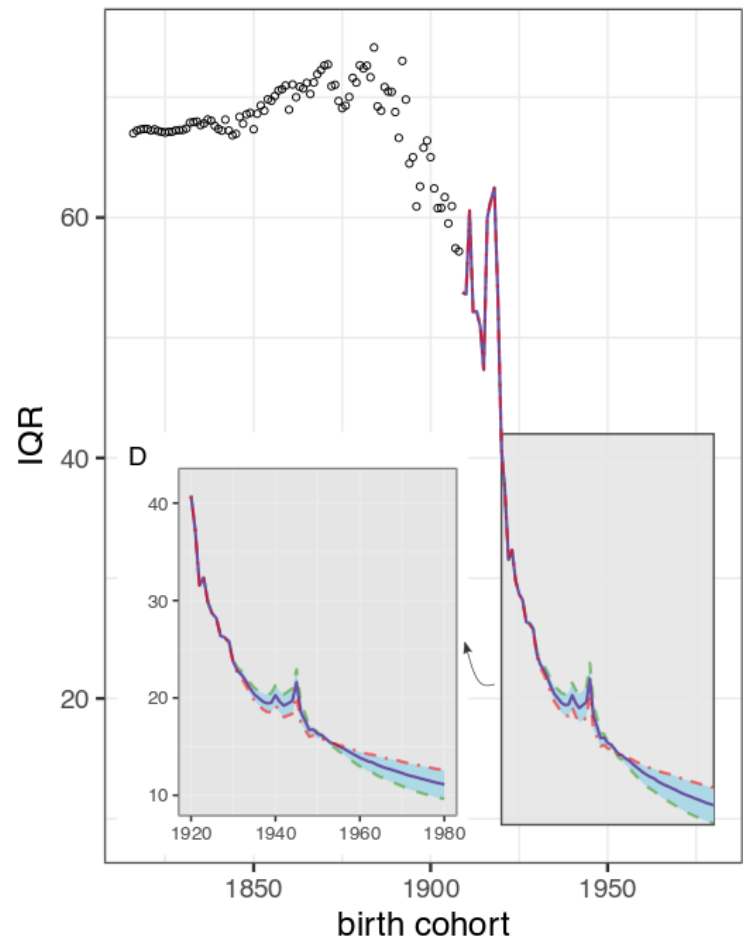

Figure A5: Panel A: Estimated $k_{t}$ for the years 1960--2018 (points) and forecast $k_{t}$ with $95 \%$ prediction intervals for the years 2019--2100 (lines) for French females, ages 0-110+. The colours highlight the median, upper and lower percentile forecasts of $k_{t}$. Panel B: Observed (points) and forecast (lines) cohort life expectancy at birth, birth cohorts 1751--1980. Panel C: Observed (points) and forecast (lines) cohort interquartile range at birth, birth cohorts 1751--1980. Panel D: Zoom in on the IQR forecasts for cohorts 1920--1980. 

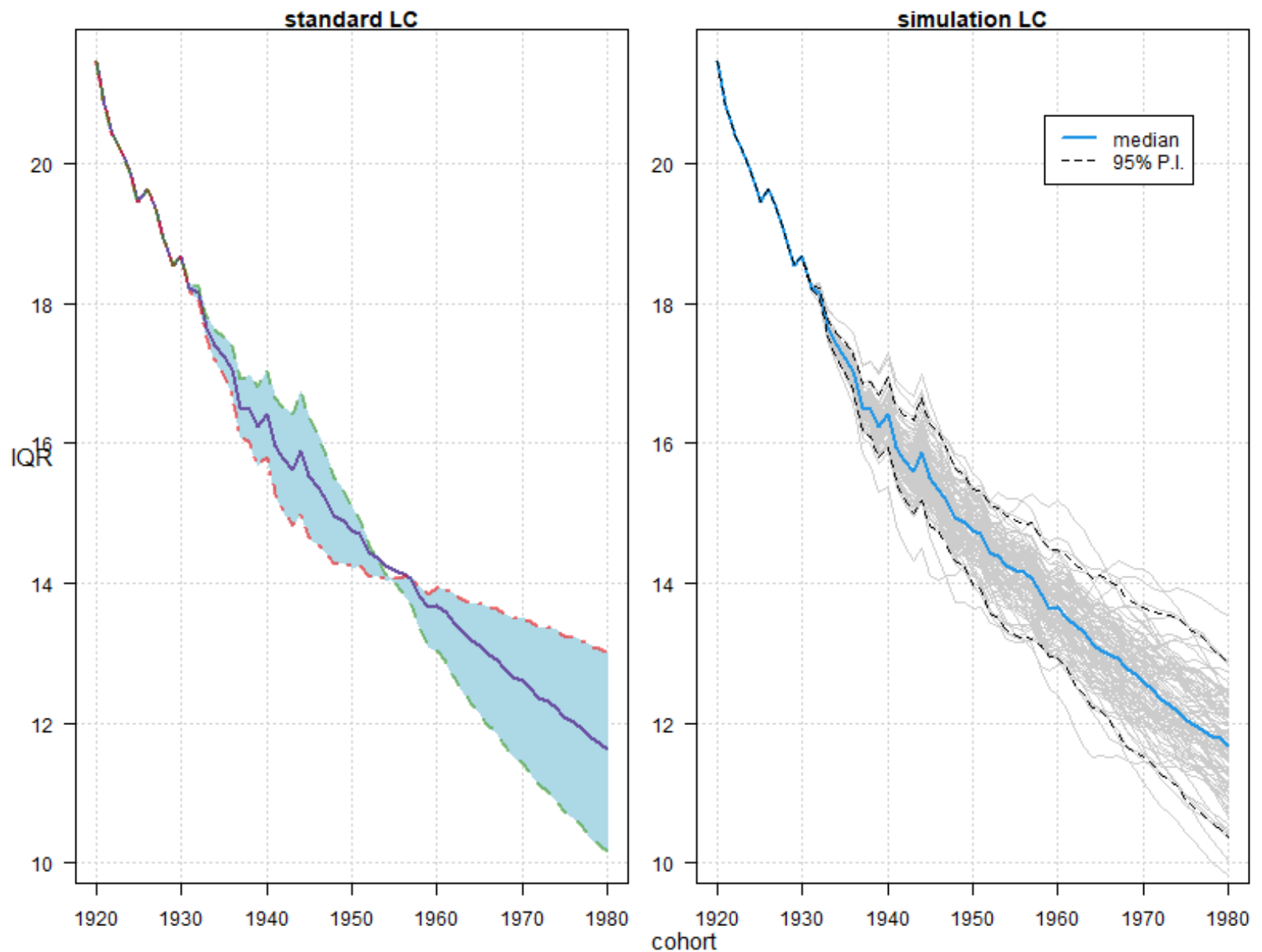

Figure A6: Comparison of forecast cohort interquartile range (IQR) derived from the two approaches of estimating prediction intervals from the LC model: estimating IQR from the mortality schedules obtained from using the upper and lower bounds of the $k_{t}$ time series parameter (left) and IQR trajectories derived from the mortality schedule corresponding to each single simulated $k_{t}$ time series (right), applied to Swedish females, ages 0-110+. To better highlight differences between the two approaches, the $x$-axis is restricted to cohorts from 1920 to 1980, as in Panel D of Figure 2. 

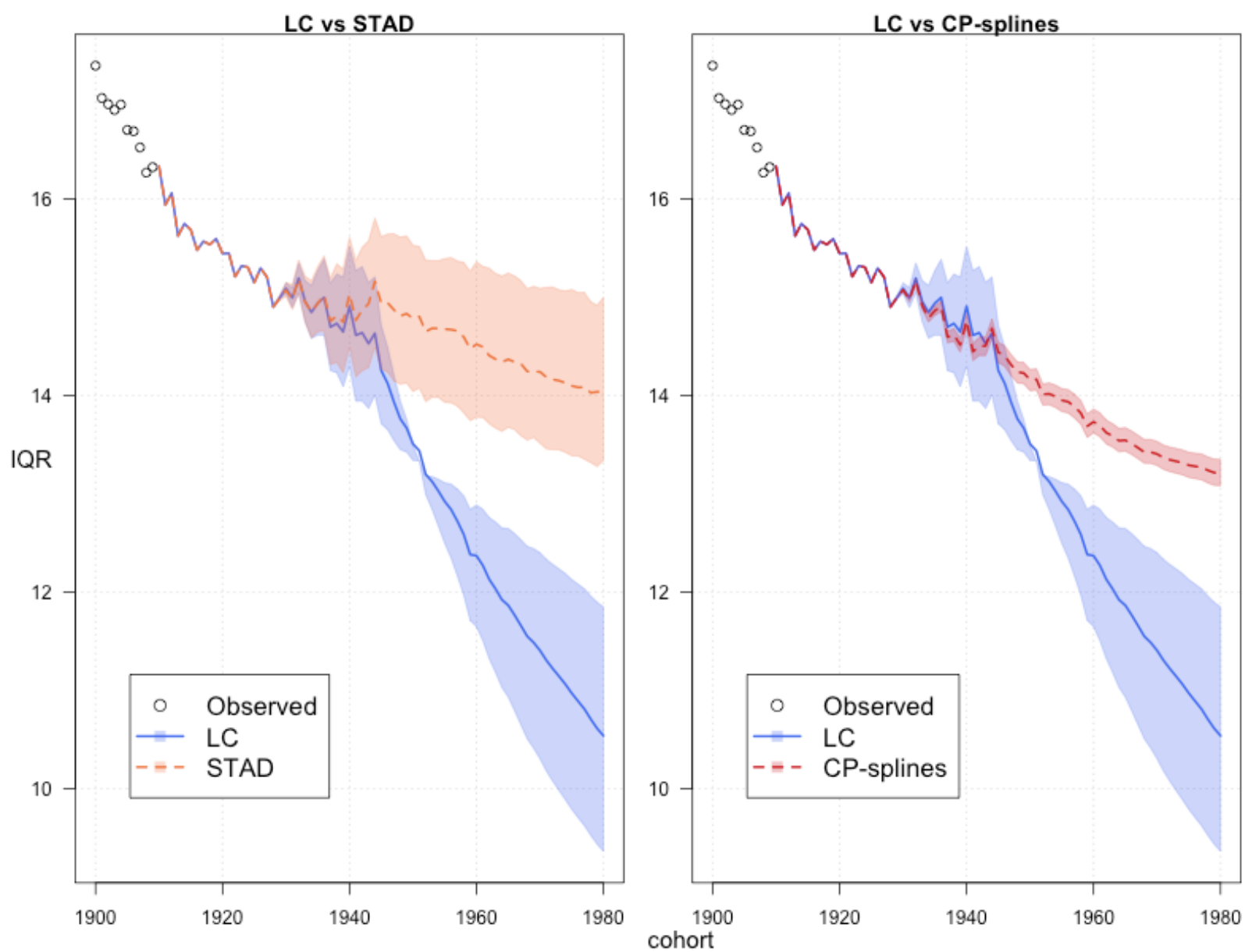

Figure A7: Results 'taking the diagonals' comparing the Lee-Carter (LC) model with the STAD period forecasting methodology (left panel) and the CP-splines approach (right panel), Swedish females, ages 30-110+. The STAD and CP-splines were selected as two methods among the few forecasting approaches that operate outside the LC framework and assumptions. To better highlight differences from the three approaches, the x-axis is restricted to cohorts from 1900 to 1980. 